\title{
Incidencia y factores asociados al abandono del tratamiento antituberculoso
}

\author{
Flor de María Cáceres ${ }^{1}$, Luis Carlos Orozco ${ }^{2}$ \\ ${ }^{1}$ Departamento de Salud Pública, Escuela de Medicina, Facultad de Salud, Universidad Industrial de \\ Santander, Bucaramanga, Colombia. \\ 2 Escuela de Enfermería, Facultad de Salud, Universidad Industrial de Santander, Bucaramanga, Colombia.
}

Introducción. La tuberculosis es un problema de salud pública. El abandono del tratamiento aumenta la morbimortalidad, continúa el contagio y genera resistencia bacteriana. Es necesario conocer la incidencia y los factores asociados al abandono para desarrollar intervenciones. Objetivo. Determinar la incidencia y los factores asociados al abandono del tratamiento antituberculoso.

Materiales y métodos. Estudio de seguimiento a una cohorte de adultos con tuberculosis, residentes en área urbana. Se definió abandono como faltar durante un mes o más al tratamiento. Los pacientes fueron entrevistados al ingreso y seguidos hasta el abandono, la muerte o el egreso del programa. La variable de salida fue tiempo al abandono. Se calcularon las tasas de abandono, las curvas de supervivencia y se elaboró un modelo de regresión de Cox para ajustar las variables asociadas.

Resultados. Se les hizo seguimiento a 261 personas, 39 (14,9\%; IC95\% 10,8-19,9) abandonaron (tasa: 0,4 episodios por 1.000 días-paciente, IC95\% 0,2-0,8). Fueron factores protectores: tener apoyo familiar (HR, hazard ratio $=0,36$, IC95\% 0,15-0,90), efectos secundarios ( $H R=0,22, I C 95 \% 0,09-0,58)$ y recibir tratamiento donde se le hizo el diagnóstico ( $H R=0,28$, IC95\% 0,12-0,63). Los factores de riesgo fueron los siguientes: inicio de síntomas mayor de dos meses (HR=14,25, IC95\% 1,80-112,7), estrato socioeconómico bajo (HR=3,90, IC95\% $2,11-9,26)$, tener entre 21 y 30 años (HR=20,61, IC95\% 2,43-175,4), haber estado detenido $(H R=2,23$, IC95\% 1,00-5,36), tener más de dos faltas al tratamiento (HR=6,62, IC95\% 2,8115,586) y tener $\mathrm{VIH} /$ sida (HR=2,94, IC95\% 1,60-5,39).

Conclusiones. El abandono es mayor que en los reportes anteriores y que en otros lugares. Se recomienda hacer diagnóstico temprano, tratamiento oportuno, fomentar el apoyo familiar, realizar intervenciones ante faltas al tratamiento, especialmente en personas con $\mathrm{VIH} / \mathrm{sida}$, de estratos bajos y con antecedentes carcelarios.

Palabras clave: Mycobacterium tuberculosis, tuberculosis/terapia, quimioterapia, terapia por observación directa, negativa del paciente al tratamiento, incidencia.

\section{Incidence of and factors for non-compliance to antituberculous treatment}

Introduction. Tuberculosis is a public health problem. Non-compliance with treatment regimes increases morbidity-mortality, perpetuates transmission and generates bacterial resistance. It is necessary to know incidence and associated factors to non-compliance for performance interventions.

Objective. The incidence of and associated factors associated with non-compliance to antituberculous treatment were investigated.

Materials and methods. A follow-up study was conducted in an adult cohort with tuberculosis, living in an urban area. Non-compliance was defined as treatment default of 30 days or more. Patients were interviewed at the initiation of treatment and and re-interviewed in subsequent intervals. Outcome was defined as the period of time until treatment abandonment. Noncompliance rates were calculated, as well as survival curves; the Cox regression model was used to adjust for associated variables. 
Results. Of the 261 patients who were interviewed, 39 (14.9\%) had abandoned treatment (rate 0.4 episodes/1,000 days-person, $95 \% \mathrm{Cl} 0.2-0.8)$. Factors associated with compliance were family support $(\mathrm{HR}=0.4,95 \% \mathrm{Cl} 0.2-0.9)$, secondary drug effects $(\mathrm{HR}=0.2,95 \% \mathrm{Cl} 0.1-0.6)$ and opportunity to receive treatment at the clinic where tuberculosis was diagnosed $(H R=0.3$, $95 \% \mathrm{Cl} 0.1-0.6)$. Risk factors for non-compliance were as follows: treatment requiring $>2$ months ( $\mathrm{HR}=14.3,95 \% \mathrm{Cl} 1.8-112.7)$, low socioeconomic status ( $\mathrm{HR}=3.90,95 \% \mathrm{Cl} 2.1-9.3)$, age between 21-30 years $(\mathrm{HR}=20.6,95 \% \mathrm{Cl} 2.4-175.4)$, history of incarceration ( $\mathrm{HR}=2.2,95 \% \mathrm{Cl} 1.0-5.4)$, skipping treatments more that twice $(\mathrm{HR}=6.6,95 \% \mathrm{Cl} 2.8-15.6)$ and co-infection with HIV/AIDS (HR=2.9, 95\% $\mathrm{Cl} 1.6-5.4)$.

Conclusion. Non-compliance rate is higher than previously reported. The data recommend the following strategies for improving compliance with antituberculosis treatment: (1) early diagnosis, (2) opportune treatment, (3) improved family support and (4) immediate intervention if a treatment is missed -especially in patients with HIV/AIDS, from low socioeconomic strata, or with record of incarceration.

Key words: Mycobacterium tuberculosis, tuberculosis/therapy, drug therapy, directly observed therapy, treatment refusal, incidence.

La tuberculosis es un problema de salud pública. Se considera que cerca de la tercera parte de la población mundial está infectada (1). El abandono o falta del cumplimiento del tratamiento se presenta cuando el paciente no asiste a recibir los medicamentos durante un mes o más (2). Actualmente, se reconoce como el más importante obstáculo para el control de la tuberculosis (3). En 1995 se informó una proporción de abandono para América del 6\% (4) y en el 2002 para Colombia del 8\% (5). En Bucaramanga, Colombia, entre 1990 y 1999 fue del $11 \%$ (6).

El abandono involucra factores del paciente, el tratamiento, el ambiente y la prestación de los servicios de salud $(7,8)$. Entre sus consecuencias están el aumento de la morbimortalidad, la probabilidad de contagio a otras personas (9) y la aparición de resistencia bacteriana (10) con el aumento del costo del tratamiento (11).

Dado que la proporción de abandono del tratamiento está por encima de la propuesta por la Organización Mundial de la Salud (menos del $5 \%$ ) (1), y que no se conocen los factores asociados, en nuestro medio, se hizo un estudio de cohortes con el objetivo de determinar las tasas

Correspondencia:

Flor de María Cáceres, Departamento de Salud Pública, Universidad Industrial de Santander, Carrera 32 № 29-31, Bucaramanga, Colombia.

Teléfono: (097) 645 4726; fax: (097) 6351646 fmcacer@uis.edu.co, flordemar200@yahoo.com

Recibido: 28/03/07; aceptado: 01/08/07 de abandono y los factores asociados. Las hipótesis fueron de asociación entre el abandono y el apoyo familiar como factor protector y ser hombre, consumir alcohol (12), tener baja escolaridad y presentar efectos secundarios como factores de riesgo.

\section{Materiales y métodos}

\section{Diseño}

Estudio observacional de seguimiento a una cohorte concurrente.

\section{Población y muestra}

Desde junio del 2002 hasta mayo del 2003 se incluyeron consecutivamente 261 personas mayores de 12 años, residentes en Bucaramanga con diagnóstico de tuberculosis e inicio del tratamiento de menos de 15 días. Los participantes debían estar en capacidad de contestar una entrevista y dar su consentimiento para realizarla. La muestra calculada fue de 258 participantes, considerando una proporción de abandono del $10 \%$ y falta de apoyo familiar en el $20 \%$ de los pacientes, con poder del $80 \%$ y una confiabilidad del $95 \%$, detectar hazard ratio (HR) de 3,0 para la asociación entre abandono y falta de apoyo familiar.

La variable de salida fue el tiempo al momento del abandono. Se averiguó por todas aquellas variables que la literatura revisada reportó como asociadas al abandono del tratamiento con el fin de establecer la presencia de confusión. 


\section{Recolección de la información}

Se elaboró y probó un formato para tal fin, al cual se le midió validez de contenido y reproducibilidad. Una enfermera entrenada, entrevistó los pacientes en la institución prestadora de salud (IPS) donde recibían el tratamiento.

Cada mes realizaba visita a la IPS, revisaba la historia clínica del paciente, se entrevistaba con la enfermera encargada del programa de tuberculosis y transcribía la asistencia diaria del paciente a recibir tratamiento, los efectos secundarios, la morbilidad, la mortalidad o el abandono. El seguimiento terminaba cuando el paciente completaba las 84 dosis de medicamentos correspondientes al esquema DOTS (directly observed therapy short-course), presentaba abandono o fallecía.

\section{Procesamiento y análisis de los datos}

Se elaboró una base de datos con doble digitación en Epi-Info 6.04b (13), se exportó al programa Stata 8.0 (14) en el cual se calcularon las medidas de tendencia central y la variabilidad para variables continuas y las proporciones para las variables nominales y ordinales. Se calcularon las tasas de abandono y las razones de tasas de abandono (IRR) con los intervalos de confianza del 95\% (IC95\%). A las variables que mostraron asociación con el abandono se les elaboró la curva de supervivencia libre de abandono de Kaplan y Meier (15), comparándolas por medio de la prueba de Wilcoxon-Breslow (16). Para el análisis multivariado se incluyeron las variables que mostraron asociación estadística con el abandono y falta de apoyo familiar, con probabilidad de error tipo 1 , valores de $p<0,2$ o que cambiaran el estimado del efecto del apoyo familiar sobre el abandono en más del $10 \%$, siguiendo las recomendaciones de Greenland (17), Christensen (18) y Grambsch y Thernau (19). Se elaboró el modelo de riesgos proporcionales (regresión de Cox) (20), siguiendo la propuesta de Cleves et al. (21). Se estimó la bondad de ajuste del modelo por medio de las estrategias de Cox y Snell (22) y el método de May y Hosmer (23). En todos los casos se consideró significancia estadística cuando los valores de $P$ eran menores de 0,05 y el IC95\% no incluía el 1.

\section{Consideraciones éticas}

El estudio fue aprobado por el Comité de Ética de la Facultad de Salud de la Universidad Industrial de Santander.

\section{Resultados}

Se siguieron 261 pacientes. En la figura 1 se observa el flujograma de los participantes en el estudio.

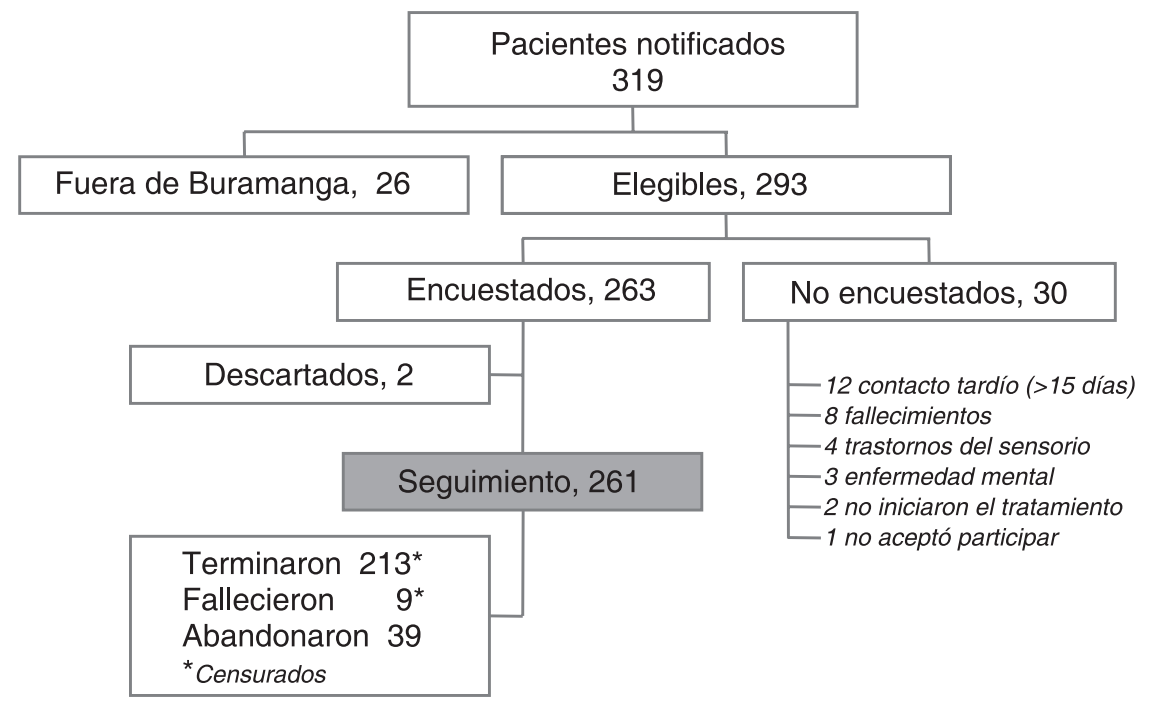

Figura 1. Flujograma de los participantes. Cohorte de tuberculosis, Bucaramanga, Colombia, 2002-2003. 
El tiempo total de seguimiento fue de 43.499 días paciente; se presentaron 39 abandonos, para una incidencia acumulada de 14,9\% (IC95\% 10,8-19,9) y una densidad de incidencia de 0,90 abandonos por mil días paciente (IC95\% 0,66-1,23).

La edad de los participantes estuvo entre 12 y 96 años, con mediana de 38; la escolaridad entre 0 y 20 años (mediana de 5 años) y el tiempo de evolución de la tuberculosis entre 0 y 348 meses (mediana de 4). En el cuadro 1 se describen otras características de los participantes.

En cuanto a las tasas de incidencia de abandono del tratamiento, las personas con apoyo familiar presentaron incidencia de abandono de 0,74 por
1.000 días paciente con intervalo de confianza del $95 \%$ entre $0,5-1,1$, quienes tenían edad entre 21 y 30 años, 1,25 (IC95\% 0,73-2,15), más de dos meses con síntomas de tuberculosis antes del diagnóstico 1,4 (IC95\% 0,8-2,3), haber estado detenido 1,7 (IC95\% 1,2-4,9), vivir en estrato bajo 1,2 (IC95\% 0,9-2,3), tener VIH-sida 1,8 (IC95\% 0,8-4,4), recibir tratamiento en la misma institución donde se hizo el diagnóstico 0,7 (IC95\% 0,2-1,0) y presentar efectos secundarios al tratamiento 0,7 (IC95\% 0,5-1,0).

En el cuadro 2 se presenta el modelo multivariado de regresión de Cox. Las variables incluidas en el modelo final cumplieron el principio de

Cuadro 1. Características de los participantes.

\begin{tabular}{|c|c|c|c|}
\hline \multicolumn{2}{|l|}{ Variable } & \multirow{2}{*}{$\begin{array}{r}\mathbf{N} \\
162\end{array}$} & \multirow{2}{*}{$\begin{array}{c}\% \\
62,1 \\
37,9\end{array}$} \\
\hline Sexo & $\begin{array}{l}\text { Hombres } \\
\text { Mujeres }\end{array}$ & & \\
\hline Raza & $\begin{array}{l}\text { Mestizos } \\
\text { Blancos } \\
\text { Afroamericanos }\end{array}$ & $\begin{array}{r}155 \\
72 \\
24\end{array}$ & $\begin{array}{r}37,9 \\
59,4 \\
9,2\end{array}$ \\
\hline Estado civil & $\begin{array}{l}\text { Soltero } \\
\text { Casado } \\
\text { Unión libre } \\
\text { Separado } \\
\text { Viudo }\end{array}$ & $\begin{array}{r}102 \\
81 \\
40 \\
30 \\
8\end{array}$ & $\begin{array}{r}39,1 \\
31,0 \\
15,3 \\
11,5 \\
3,1\end{array}$ \\
\hline Régimen de seguridad social & $\begin{array}{l}\text { Contributivo } \\
\text { Subsidiado } \\
\text { Vinculado } \\
\text { Ninguno }\end{array}$ & $\begin{array}{r}102 \\
52 \\
82 \\
25\end{array}$ & $\begin{array}{r}39,1 \\
19,9 \\
31,4 \\
9,6\end{array}$ \\
\hline Estrato de la vivienda & $\begin{array}{l}\text { Cero } \\
\text { Uno } \\
\text { Dos } \\
\text { Tres } \\
\text { Cuatro a seis }\end{array}$ & $\begin{array}{r}7 \\
50 \\
119 \\
61 \\
24\end{array}$ & $\begin{array}{r}2,7 \\
19,2 \\
45,6 \\
23,4 \\
9,2\end{array}$ \\
\hline Tiene empleo & & 96 & 35,6 \\
\hline $\begin{array}{l}\text { Tiene conocimientos } \\
\text { sobre la tuberculosis }\end{array}$ & $\begin{array}{l}\text { Ningún conocimiento } \\
\text { Conocimiento deficiente } \\
\text { Regular } \\
\text { Bueno } \\
\text { Excelente }\end{array}$ & $\begin{array}{r}28 \\
65 \\
101 \\
58 \\
9\end{array}$ & $\begin{array}{r}10,7 \\
24,9 \\
38,7 \\
22,2 \\
3,4\end{array}$ \\
\hline \multirow{3}{*}{\multicolumn{2}{|c|}{$\begin{array}{l}\text { Ha estado detenido alguna vez en la vida } \\
\text { Considera que la familia lo apoya } \\
\text { Reciben tratamiento en institución prestadora de salud } \\
\text { donde se diagnosticó la tuberculosis }\end{array}$}} & 64 & 24,5 \\
\hline & & 224 & 86,0 \\
\hline & & 180 & 69,0 \\
\hline \multicolumn{2}{|c|}{ Presentó efectos secundarios al tratamiento } & 207 & 79,3 \\
\hline \multicolumn{2}{|c|}{ Presentó faltas al tratamiento } & 119 & 45,6 \\
\hline \multicolumn{2}{|l|}{ Terminó el tratamiento } & 213 & 81,6 \\
\hline \multicolumn{2}{|l|}{ Abandonó el tratamiento } & 39 & 14,9 \\
\hline
\end{tabular}


Cuadro 2. Variables asociadas al abandono del tratamiento antituberculoso. Modelo de regresión de Cox.

\begin{tabular}{lrrr}
\hline Variable & \multicolumn{1}{c}{ Hazard ratio (IC95\%) } & $\mathbf{p}$ \\
\hline Tener edad: $12-20$ años & 6,56 & $(0,36-118,5)$ & 0,202 \\
$21-30$ & 20,61 & $(2,43-175,4)$ & 0,006 \\
$31-40$ & 3,93 & $(0,46-33,77)$ & 0,213 \\
$41-50$ & 8,00 & $(0,93-68.57)$ & 0,058 \\
$51-60$ & 5,29 & $(0,58-48,65)$ & 0,141 \\
61 y más años & Referente &.-- \\
Contar con apoyo familiar & 0,36 & $(0,15-0,90)$ & 0,028 \\
Vivir en estrato bajo & 3,90 & $(2,11-9,26)$ & 0,025 \\
Tener más de dos meses con síntomas antes de realizar el diagnóstico & 14,25 & $(1,80-112,7)$ & 0,012 \\
Tener VIH-sida & 2,94 & $(1,60-5,39)$ & 0,000 \\
Haber estado detenido & 2,23 & $(1,00-5,36)$ & 0,059 \\
Recibir tratamiento en donde se diagnosticó & 0,28 & $(0,12-0,63)$ & 0,002 \\
Presentar efectos secundarios al tratamiento & 0,22 & $(0,09-0,58)$ & 0,002 \\
Presentar más de dos faltas al tratamiento & 6,62 & $(2,81-15,58)$ & 0,000 \\
\hline
\end{tabular}

IC95\%: intervalo de confianza del 95\%

proporcionalidad del riesgo y el modelo mostró un ajuste adecuado.

\section{Discusión}

La principal debilidad de este estudio consiste en que se hizo énfasis en factores relacionados con el paciente y no se profundizó en aspectos de la prestación de los servicios de salud. La calidad de la atención se midió por la percepción del paciente, no se estudiaron barreras de acceso a los servicios, ni calidad de la atención por otros métodos.

Con respecto al tamaño de la muestra, el número de eventos y la relación con el número de variables introducidas en el modelo final, se considera que no se presentó ningún tipo de error; dado que el modelo presentó un adecuado ajuste y que inclusive, recientes publicaciones sustentan la posibilidad de disminuir el número de eventos por variable en el modelo, obteniendo un adecuado control de la confusión (24). Por lo anterior, este hecho no se considera una debilidad del estudio.

La fortaleza radica en que es uno de los pocos estudios de cohortes de pacientes con tuberculosis en el que se hizo seguimiento, se calculó tanto la proporción de abandono como la densidad de incidencia y se realizó análisis multivariado de regresión de Cox para maximizar la potencia del estudio (25).

La incidencia de abandono encontrada fue de $14,9 \%$, está por encima de reportes previos: 6,8 y $11 \%$, respectivamente $(4,5,6)$. Una explicación podría ser que el problema ha aumentado, producto de los cambios ocurridos en la dinámica del programa como consecuencia de la reforma del sector salud en el país, que pasó de ser administrado por el Estado para ser delegado a empresas contratistas (26). Este nuevo esquema afectó la visión colectiva de las actividades del control de la tuberculosis (27).

En cuanto a los factores asociados, se confirma que contar con apoyo familiar es factor protector. Otro factor protector fue recibir el tratamiento en la misma institución donde se hizo el diagnóstico. Este hecho puede ser explicado porque los pacientes que se tienen que desplazar a otras instituciones para que se les realice el diagnóstico y regresar a la institución de origen sufren las fallas en la coordinación de las acciones del programa y la falta de integración en la prestación de los servicios, tal como fue reportado en un estudio en Italia (28). Significa que se debe fortalecer la búsqueda activa de tuberculosis entre sintomáticos respiratorios, hacer diagnóstico precoz y tratamiento oportuno en la misma institución para mejorar el cumplimiento del paciente al tratamiento.

La presentación de efectos secundarios estuvo asociado como factor protector. Este aspecto ha sido reportado como factor de riesgo (29). Es posible que en esta cohorte quienes presentaron efectos secundarios percibieran la enfermedad 
como más grave y, por lo tanto, persistieran recibiendo el tratamiento. Se requiere más estudios para mejorar la evidencia en este aspecto.

Entre los factores de riesgo se encontraron haber tenido demora en el diagnóstico, vivir en estrato socioeconómico bajo, tener entre 21 y 30 años, haber estado detenido, presentar más de dos faltas al tratamiento y ser portador del VIH/sida.

Faltar en más de dos oportunidades a recibir los medicamentos aumentó la velocidad de abandono. Este hallazgo orienta acciones de prevención pues una vez que falte el paciente, se debe iniciar su ubicación y seguimiento, estrategia que se encontró que era efectiva en un estudio realizado en Camboya (30).

Algunas variables reportados como factores de riesgo en otros estudios, tales como ser hombre, ser soltero o estar viudo o separado, no tener empleo, no contar con vivienda propia y tener un bajo nivel educativo, no se asociaron como factores de riesgo para el abandono del tratamiento en el presente estudio. Las razones pueden ser que esas condiciones sean diferentes en nuestra población o que hayan diferencias en la definición de las mismas, en el presente estudio.

Se recomienda profundizar sobre el papel que desempeñan los servicios de salud en el abandono del tratamiento contra la tuberculosis; usar diseños experimentales para probar la eficacia de intervenciones que mejoren el cumplimiento terapéutico; posiblemente, el uso de los diagnósticos de enfermería de la North American Nursing Asociation, es decir, incumplimiento del tratamiento y manejo inefectivo del esquema terapéutico (31), con intervenciones específicas y medición de resultados, permitan mejorar el cumplimiento del tratamiento contra la tuberculosis, aspecto de gran importancia para la salud pública.

\section{Financiación}

Estudio cofinanciado por el Colciencias y la Universidad Industrial de Santander, mediante el contrato No.101-2003; código 1102-04-12907.

\section{Conflicto de intereses}

Los autores no tenemos conflicto de intereses.

\section{Referencias}

1. Organización Mundial de la Salud. Informe sobre tuberculosis en América. [Consultado: noviembre de 2001]. http://www.who.int/inffs/en/fact104.html.

2. Ministerio de Salud. Dirección General de Promoción y Prevención. Guía de atención de la tuberculosis pulmonar y extrapulmonar. Bogotá: Ministerio de Salud; 2001. p.1-47.

3. Pablos-Méndez A, Cnirsch CA, Barr RG, Lerner BH, Frieden TR. Nonadherence in tuberculosis treatment: Predictors and consequences in New York City. Am J Med. 1997;102:164-70.

4. World Health Organization Global. TB Programme. Report of the Ad Hoc Committee on the Tuberculosis Epidemic. WHO/TB/98.245. Geneva: World Health Organization; 1998.

5. Chaparro PE, García I, Guerrero MI, León CI. Situación de la tuberculosis en Colombia, 2002. Biomédica. 2004;24(Suppl.1):102-14.

6. Cáceres FM, Flórez NS, García ME, Ortega N, Téllez LE. Características epidemiológicas de los pacientes con tuberculosis en Bucaramanga, 1990 a 1999. Médicas UIS. 2001;15:89-98.

7. Homedes N, Ugalde A. ¿Qué sabemos del cumplimiento de los tratamientos médicos en el tercer mundo? Bol Of Sanit Panam. 1994;116:491-517.

8. Jaramillo E. Pulmonary tuberculosis and healthseeking behavior: How to get a delayed diagnosis in Cali, Colombia. Trop Med Int Health. 1998; 3:138-44.

9. Pozsik C. Compliance with tuberculosis therapy. Med Clin North Am. 1993;77:1289-301.

10. Eraker SA, Kirscht JP, Becker MH. Understanding and improving patient compliance. Ann Intern Med. 1984;100:258-68.

11. Singh $\mathbf{M}$. Adherence to anti-tuberculosis treatment. Indian Pediatr. 1999;36:1285-6

12. Orozco LC, Hernández R, de Usta CY, Cerra M, Camargo D. Factores de riesgo para el abandono (no adherencia) del tratamiento antituberculoso. Médicas UIS. 1998;12:169-72.

13. Dean J, Dean DA, Coloumbier D, Brebdel KA, Smith DC, Burton AH, et al. Epi Info 6.04c. A word processing, database, and statistic program for public health. Atlanta: Center for Disease Control and Prevention, World Health Organization; 1997

14. STATA Corporation Inc. STATA 8.0. College Station. Texas: STATA Corporation Inc; 2003.

15. Matthews DE, Farawell VT. Curvas de sobrevida. En: Estadística médica: aplicación e interpretación. 2 edición. Barcelona: Editorial Salvat; 1990.p.132-48.

16. Green MS, Symons MJ. A comparison of the logistic risk function and the proportional hazards model in 
prospective epidemiologic studies. J Chronic Dis. 1983;36:715-24

17. Greenland $\mathbf{S}$. Modeling and variable selection in epidemiologic analysis. Am J Public Health. 1989; 79: 340-9.

18. Christensen E. Multivariate survival analysis using Cox's regression model. Hepatology. 1987;7:136-58.

19. Grambsch PM, Therneau TM. Proportional hazard test and diagnostics based on weighted residuals. Biometrika. 1994;81:515-26.

20. Cox DR. Regression models and life-tables (with discussion) J R Stat Soc. 1972;34B:187-220.

21. Cleves MA, Gould WW, Gutiérrez RG. An Introduction to survival analysis using stata. Texas: Stata Press; 2002.

22. Cox DR, Snell EJ. A general definition of residuals (with discussion). J R Stat Soc. 1968;30B:248-75.

23. May S, Hosmer DW. A simplified method for calculating a goodness-of-fit test for the proportional hazard model. Lifetime Data Anal. 1998;4:109-20.

24. Vittinghoff E, McCulloch CE. Relaxing the ruler of ten events per variable in logistic and Cox regression. Am J Epidemiol. 2007;165:710-8.
25. Stare J, O`Quigley J. Fit and frailties in proportional hazard regression. Biometrical J. 2004;46:157-64.

26. Arbeláez MP, Gaviria MB, Franco A, Restrepo R, Hincapié D, Blas E. Tuberculosis control and managed competition in Colombia. Int $\mathrm{J}$ Health Plann Manage. 2004;19(Suppl.1):S25-43.

27. Carvajal R, Cabrera GA, Mateus JC. Efectos de la reforma en salud en las acciones de control de tuberculosis en el Valle del Cauca, Colombia. Biomédica. 2004;24(Suppl.1):138-48.

28. Moro ML, Resi D, Lelli B, Nicoli A, Gaglioti C, Falcone F. Barriers to effective tuberculosis control: a qualitative study. Int J Tuberc Lung Dis. 2005;9:1355-60.

29. Ferrer X, Kirschbaum A, Toro J, Jadue J, Muñoz M, Espinoza A. Caracterización del paciente tuberculoso que abandona el tratamiento, Santiago de Chile. Bol Oficina Sanit Panam. 1991;111:423-31.

30. Kimerling ME, Petri L. Tracing as part of tuberculosis control in a rural Cambodian district during 1992. Tuberc Lung Dis. 1995;76:156-9.

31. North American Nursing Diagnosis Association (NANDA). Nursing diagnoses: definitions and classification 2001-2002. Barcelona: Ediciones Harcourt; 2001. 